\title{
Ganz einfach online fortbilden: CME-Kurse auf conrad
}

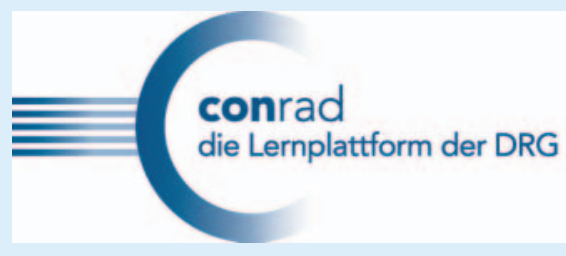

Auf conrad, der digitalen Lernplattform der Deutschen Röntgengesellschaft, finden Sie ein vielfältiges Angebot an CME-anerkannten Kursen. Die Themen reichen von der Neuroradiologie über die muskuloskeletta- le Radiologie hin zur onkologischen Bildgebung. Auch Inhalte aus der Gastrointestinal- und Abdominaldiagnostik werden zur Weiterbildung angeboten.

Eines haben alle Kurse gemeinsam: Sie enthalten Auszüge der am besten evaluierten Vorträge des RÖKO DIGITAL 2020 und des 102. Deutschen Röntgenkongresses, der im vergangenen Jahr stattfand. Neben den Videomodulen stehen Ihnen Fragen zur Lernerfolgskontrolle zur Verfügung. Nach
Abschluss eines Kurses ist es Ihnen möglich, eine Teilnahmebescheinigung herunterzuladen, die Sie dann bei der Landesärztekammer einreichen können.

Mit der Bearbeitung dieser Kurse werden jeweils zwei beziehungsweise vier CMEPunkte vergeben.

Überzeugen Sie sich selbst von unserem Angebot: www.conrad.drg.de. 